\title{
A Software Architecture to Facilitate the creation of DRM Systems
}

\author{
Koen Buyens, Sam Michiels, and Wouter Joosen \\ IBBT-DistriNet research group, Department of Computer Science, \\ K.U.Leuven, Celestijnenlaan 200A, B-3001 Leuven, Belgium \\ \{koen.buyens,sam.michiels\}@cs.kuleuven.be \\ T: ++3216327700 - F: ++3216327996
}

\begin{abstract}
Although various publications confirm the need for a generic DRM software architecture, we observe that current efforts to define a DRM architecture do not always provide sufficient support to enable the creation and management of DRM systems and content distribution applications. This is a considerable problem that implies a crucial challenge for the evolution of DRM, given the impact of a software architecture on the functional and non-functional qualities of the implementation. This paper (1) presents a generic DRM architecture, (2) evaluates it in the context of interoperability, extendability, and modifiability, and (3) compares it to related work in the Digital Media Project (DMP). To the best of our knowledge, the proposed architecture is more detailed than related work published so far.
\end{abstract}

\section{INTRODUCTION}

Software architecture technology is playing an increasingly important role in the development and evolution of DRM systems. The DRM community has confirmed the importance of a generic software architecture that guides developers and that enables the reuse of third party components [1], [4], [5], [7]-[11].

Reflecting on current state-of-the-art DRM technologies, we observe three common characteristics [9], [10]. First of all, the majority of DRM technologies implement a common set of core services concerning content handling, licensing, and access control. In an earlier publication we have studied the main DRM systems, thereby identifying these core service components. Secondly, state-of-the-art DRM technologies extend the core functionality with a wide variety of services, such as consumer tracking, payment, or abuse identification. And thirdly, the overall domain of DRM is rapidly evolving with respect to security technologies, licensing policies, and payment schemes.

These observations raise three important challenges that have impact on consumers, producers and publishers of DRM protected content. First of all, there is need for a standardized core architecture that can be reused by content publishers to facilitate integration of DRM in their content distribution platforms. Secondly, an extended architecture, which offers DRM services beyond the core functionality, must offer a rich set of variation points in order to support varying and diverse requirements of content consumers, producers and publishers. Thirdly, given the rapidly evolving character of
DRM, interoperability, modifiability, and extendability are crucial architectural concerns for DRM.

The contribution of this paper is the presentation of a DRM software architecture that handles these three key challenges. The architecture has been developed in the context of a research project which included both consumers, producers, and a publisher of a digital news paper [12]. It is based on stateof-the-art architectural patterns which make the architecture easier to understand, extend and modify. The paper evaluates the architecture and compares it with ideas from the Digital Media Project (DMP) [5].

The remainder of this paper is structured as follows. Section II selects and defines the main quality requirements that are relevant for DRM system. Section III presents a high level overview of three key component groups and zooms in on the architecture from three perspectives: basic content handling, consumer tracking, and content securing. Section IV evaluates the proposed architecture and compares it to related work in the Digital Media Project. Conclusions are formulated in section $\mathrm{V}$.

\section{REQUIREMENTS}

This section selects and defines the main quality requirements that are relevant for DRM systems.

A quality requirement is a property of a work product (or software architecture) by which stakeholders can judge its quality. These properties, like for instance interoperability, modifiability, or extendability may have significant impact on the architecture of a system [2]. As such, quality attributes are generally accepted to be crucial attention points during the early phases of system development, i.e. analysis and architecture design.

It should be clear that typical software quality requirements must be supported when building and deploying DRM systems or content distribution applications: interoperability, modifiability, extendability, usability, testability, availability, security, scalability, performance, etc. Yet, in the context of this paper we limit the discussion to the following key architectural drivers: interoperability, modifiability, and extendability. They were chosen because we focussed on support for the rapidly evolving DRM field. Since many interpretations of these requirements exist, we provide a definition for each and motivate why it is an important concern for DRM systems. 
- Interoperability is the ability of two or more entities from different vendors to cooperate at runtime. Interoperability between DRM service components is highly important given the complexity and extensiveness of DRM functionality, as well as the rapidly evolving field, which imply that multiple variants of the same service will coexist and should be able to co-operate. By consequence, consumers must be able to obtain and consume content and licenses from any content publisher. Producers should prepare content and rights only once and be able to choose between various publishers to distribute their content. Publishers must be able to replace service components with similar components from different vendors.

- Modifiability is the ease with which a system can accommodate changes to its software. The behavior of the publishing system must be modifiable to accommodate varying business policies for licensing, payment, and rights enforcement. For instance, a publisher must be able to change its payment model from pay-per-view to a monthly subscription. The consumer should be able to modify the behavior of their DRM Client in order to submit anonymous consumption statistics instead of privacy sensitive data.

- Extendability is the ease to add new features to the system. Since the domain of software security and DRM is highly dynamic, DRM systems must embrace change and be open to extend their functionality with new or improved components. Consumers and publishers, for instance, must keep their system up to date by dynamically adding security updates or extra features.

\section{ARchitecture}

Supporting these requirements is not a trivial task to achieve. This section first presents a high level overview of a DRM architecture by identifying three coherent collections of components and explaining the interactions between them. It also shows how two key issues can be addressed by applying architectural patterns. Secondly, it introduces the main components by zooming in on the architecture from three perspectives: content handling, consumer tracking, and content securing. Details about component interfaces and representative use cases have been described in a technical report [13].

\section{A. Component groups}

A component group represents a coherent collection of components that offers a key functional aspect of DRM. We identify three component groups that represent (1) DRM services, (2) content representation and interpretation (RI), and (3) security algorithms. Figure 1 illustrates the relationships between component groups at consumer, producer, and publisher side. Since a DRM architecture is embedded within a publishing context, functionalities related to user, content, or input management ${ }^{1}$ can sometimes be reused. ${ }^{2}$

\footnotetext{
${ }^{1}$ Input management is a form of content handling and consists of classifying input submitted to the DRM system, etc.

${ }^{2}$ Text between parentheses indicates to what extent the component group reuses components from the publishing context (Context) or not (DRM).
}

- Service component group (DRM and Context): This component group offers the main services of a DRM system: content handling, licensing, access control, and input management. Some of these services can be reused from the content distribution platform at the publisher side.

- RI component group (DRM): This component group is used to represent content and licenses (described by a rights expression language (REL)), to collect information about content consumption, and to interpret policies, for instance for licensing. It contains the Representation Component, the Logging Component, and the Interpretation Component.

- Security component group (DRM): This component group provides various security algorithms to protect content against abuse, by encrypting, signing, or watermarking. These components are used by the RI component group, for instance, to enforce a licensing policy.

The architecture handles the following two key issues. First of all, it supports transparent message interception to support consumer tracking. In order to allow consumer tracking at the publisher side, consumer requests should be intercepted by the publishing system before they are handled by the publishing service component group. Yet, at the consumer side, user requests should be intercepted before reaching the consumer security component group. By consequence, the architecture should enable message interception at various places. In addition, since consumer tracking is a non-functional DRM concern that may not always be present, message interception must be transparent for all service components.

Secondly, since content cannot always be processed in one pass through the security component group, the architecture must enable cyclic processing in a controllable way. It is unnatural to structure component groups as a layered system with a sequential interaction flow [7]. Content may consist of multiple parts, each of which can have a different license associated with it resulting in different ways of protection. Think, for instance, of a video stream with subtitles in multiple languages and extra bonus tracks that can only be played after a certain date. The RI component group knows how composed content is represented and, by consequence, how these parts are glued together and protected (e.g. by the usage of the MPEG-21 REL). The security component group, however, cannot distinguish between various content items with different security requirements.

The two aforementioned issues - the need for transparent consumer tracking and non-sequential content traversal through the system - can be solved at the architectural level by applying the broker architectural style [3]. An architectural style expresses a fundamental structural organization schema for software systems. It provides a set of predefined subsystems, specifies their responsibilities, and includes rules and guidelines for organizing the relationships between them.

Three architectural patterns are useful for transparently intercepting messages and dispatching them in various ways: router, broker, and blackboard (see also Figure 2). A common 


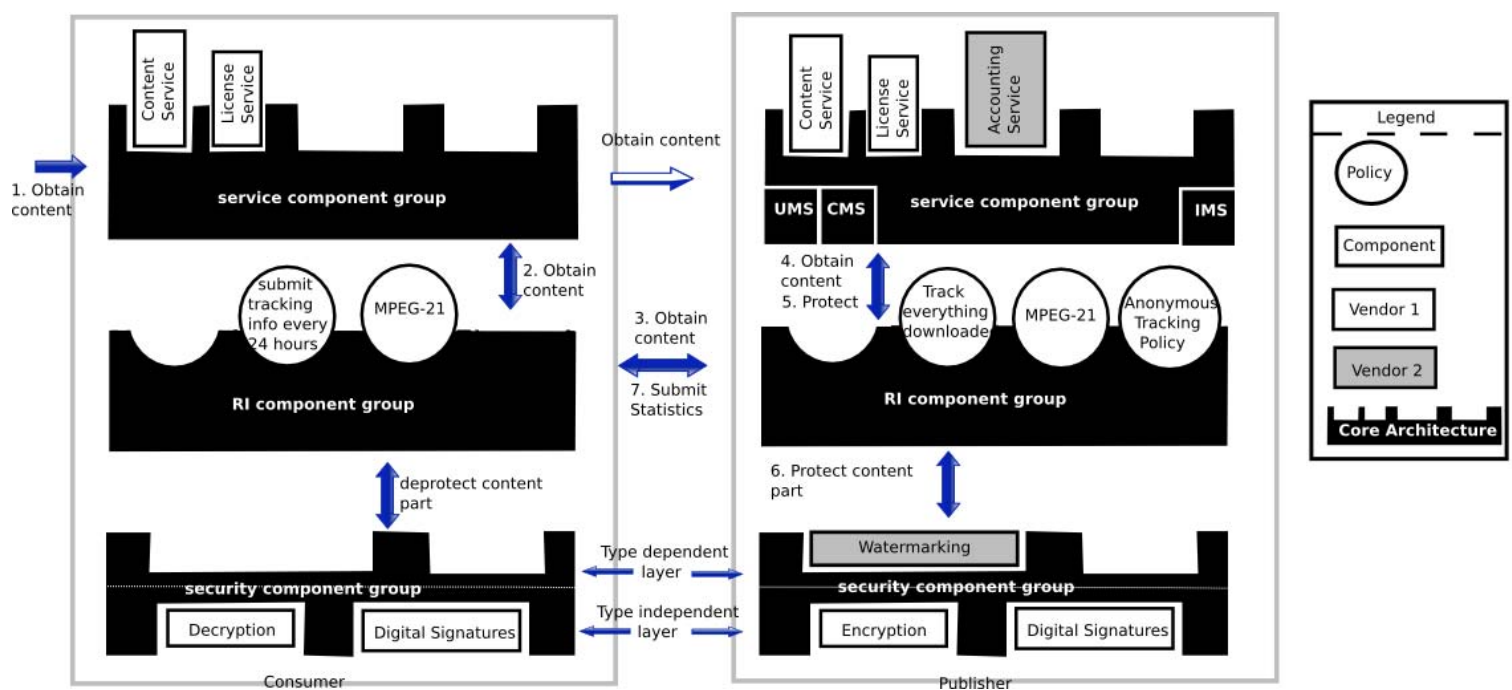

Fig. 1. Architectural overview that illustrates two main roles, consumer and publisher, which both consist of three customizable component groups. The service component group contains core publishing services (UMS, CMS and IMS) and pluggable DRM service components (License Service, Content Service and Accounting Service). The grey and white components show services provided by different vendors which illustrates interoperability between similar service components. The RI component group is used to represent content and licenses, and to interpret business policies to change the system's behavior. It acts as a dispatcher that delegates requests according to, for instance, a consumer tracking policy. The security component group provides components with algorithms for encryption/decryption, watermarking and attaching digital signatures to content. The system can easily be extended by adding components and policies.

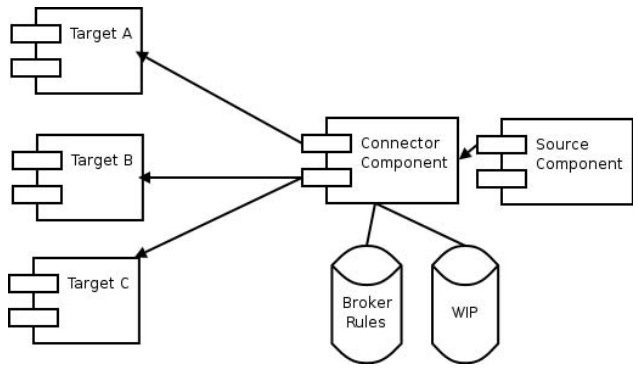

Fig. 2. The router, broker, and blackboard architectural patterns are used for transparently intercepting messages and dispatching them in various ways. The Source Component initiates an interaction that is intercepted and forwarded to zero, one, or more target components $(A, B, C)$. Selection of the Target Component is controlled by distribution rules (Broker Rules) in the connector component. Intermediate results are stored in a Work In Progress (WIP) component.

characteristic shared by these patterns is that they ensure that components never interact directly with each other, but always through a connector. The connector is called router, broker, or blackboard, depending on the role it plays in the pattern. In the router pattern, source components initiate an interaction that is routed to at most one of multiple target components. The selection of the target component is controlled by distribution rules. The broker extends the router pattern with the ability to decompose and reassemble incoming messages. Intermediate results are stored in e.g. a Work In Progress (WIP) component. In the blackboard pattern, different components make use of a shared data structure which encapsulates state data and triggers components when state has changed. Individual components make changes to state information in the blackboard, thereby incrementally producing a solution to the problem at stake.
The choice for the broker pattern is justified for three reasons: (i) tracking is possible, because interception and modification of messages is supported, (ii) new components can be easily added, and (iii) the broker can apply various business policies, such as dynamically converting content or licenses for different platforms (e.g. mobile phones and personal computers) or standards. One should note that the broker can become a bottleneck and a single point of failure, because it intercepts all communication. The blackboard, however suffers from more drawbacks, since every component should use the same representation, which makes it difficult to change data representations.

\section{B. Architecture perspectives}

We now zoom in on the architecture from three perspectives. The content handling perspective focuses on the core components of a DRM system, the consumer tracking perspective shows how the architecture can be extended with logging functionality, and the security perspective closes the content publishing loop by illustrating how content can be secured by various techniques.

Each perspective can be seen as a filter that focuses on a particular level of functionality. The lowest level introduces the core functionality that is needed for distributing content. The other levels extend this functionality with tracking and security. The combination is a full-blown architecture which supports creation, distribution and consumption of DRMprotected content, distribution of licenses, and user tracking. For each perspective, the main components are briefly introduced.

1) Content handling perspective: This perspective presents all services related to content distribution and manipulation. 
Relevant components are located in the service and the RI component groups.

Service component group. In a previous paper we have identified seven key DRM service components for licensing, access, content handling, abuser identification, content importing, consumer tracking, and payment. DRM developers should not reinvent the wheel and reuse existing components when possible. For instance, a publishing architecture typically provides support for content handling, access, and payment. By consequence, components such as the Content Service, the User Management System and the Billing System can be easily reused, possibly by adapting some policies.

RI component group. The RI component group contains a Representation Component, which is responsible for creating content in a certain representation and for converting content into another representation. This component can be used, for instance, to convert a representation from MPEG-2 to MPEG21 [14].

2) Tracking Perspective: Usage statistics can be collected at the publisher side or at the consumer side. The presented solution is similar for both sides. The following components in the service and RI component groups offer support for consumer tracking.

Service component group. The Accounting Service uses the information that the Logging Component has collected in order to produce overviews of statistical information, e.g. to provide a monthly consumption bill.

RI component group. The dispatcher architectural pattern can be applied to implement the functionalities of this component group using the following four components:

- Policy Engine: interprets policies. At the consumer side, for instance, a policy represents the license described in the MPEG-21 REL.

- Dispatcher Component: dispatches the message to the appropriate component group.

- Logging Component: logs and sends certain messages to the Tracking Component.

- Representation Component: this component has been explained above.

Each internal RI component registers itself with the Dispatcher Component and informs the Dispatcher Component with the messages it is interested in. The Dispatcher Component consults a list of delivery rules in the Policy Engine to decide how messages must be handled or logged. The main advantages of using the dispatcher pattern are (i) flexibility, (ii) ability to accommodate future developments easily by just plugging in a new module, (iii) possibility to add context in different incremental steps and (iv) to let each module be hosted by a third party. However, this approach may imply overhead and a single point of failure.

3) Security Perspective: Different protection schemes are possible, depending on the capabilities of the publishing system and the available security services. Relevant components are located in the security component group.

Security component group. The security component group contains various components, which implement algorithms and techniques for content securing, such as watermarking, fingerprinting, secure data enveloping, secure communications, certificate management, secure storage, secure timing, secure localization, key exchange, key generation, digital signatures, key management, encrypting, and key storage. These components are organized in layers: the type dependent layer contains components with content type dependent algorithms (e.g. watermarking, etc.), while the type independent layer contains components with type independent algorithms (e.g. encryption, digital signatures, etc.). The type dependent layer can use the type independent layer (e.g. encryption of a watermark).

These components are managed by an Orchestrator Component, which acts as broker/dispatcher. Advantages of applying the broker pattern in this case are (i) easy integration with existing security components, (ii) extendability: new security components can be added very easily, and (iii) flexibility: different configurations of security components are possible. Drawbacks are that (i) communication messages must be standardized, (ii) unprotected data is sent in the clear, and (iii) much communication is needed to protect a simple content part. The Representation Component consults this component group repeatedly for constructing secured content.

\section{EVALUATION}

This section sketches how the presented architecture offers support to achieve interoperability, modifiability, and extendability. After summarizing the main advantages, the architecture is compared with related work in DMP.

\section{A. Discussion}

Table I summarizes to what extent the proposed architecture supports each requirement by using well-defined service components and the broker architectural pattern.

Interoperability. The broker pattern supports interoperability, because it can automatically convert message representations. By consequence, different components can communicate with each other. The fine granularity of service components increases reusability and interoperability. Interoperability is also achieved by identifying the main DRM services as architectural components, documenting them with well-defined interfaces, and localizing them in an overall architecture.

Modifiability. Modifiability of the system's behavior to application or business specific policies is supported by the broker, in which policies can be injected. The behaviour is described in pluggable policies.

Extendability. Extendability is achieved by enabling to add security components by simply registering them at the broker which transparently maps consumer requests and business policies onto the available set of security algorithms and techniques. This pattern also orchestrates the collaboration of old security components and new ones and by consequence enhances the extendability.

\section{B. Relation to DMP}

The Digital Media Project (DMP [5]) strongly focuses on interoperability, which is clearly a very important concern for 


\begin{tabular}{|l||c|c|}
\hline Requirements & Architectural style & Advantage \\
\hline \hline Interoperability & $\begin{array}{c}\text { broker, service } \\
\text { components }\end{array}$ & $\begin{array}{c}\text { reusable service components, } \\
\text { message conversion, } \\
\text { clearly defined interfaces }\end{array}$ \\
\hline Modifiability & $\begin{array}{c}\text { broker, service } \\
\text { components } \\
\text { behavior described } \\
\text { in pluggable policies }\end{array}$ \\
\hline Extendability & $\begin{array}{c}\text { broker, service } \\
\text { components }\end{array}$ & $\begin{array}{c}\text { security components } \\
\text { registered at broker }\end{array}$ \\
\hline
\end{tabular}

TABLE I

OVERVIEW OF MAIN REQUIREMENTS, THE ARCHITECTURAL STYLES THAT SUPPORT THEM, AND THE BENEFITS THEY OFFER.

DRM. The DMP architecture defines users (e.g. consumers, producers, or publishers) as entities that perform so-called primitive functions, which represent the underlying DRM services that handle digital content. The primitive functions can be related to the three component groups identified in the architecture proposed in this paper.

Assessment of the DMP architecture by the presented requirements leads to the following conclusions. DMP achieves interoperability within a single value chain by offering finegrained primitive functions with clearly defined interfaces. Multiple primitive functions from different vendors can be composed into so-called tools that run at the consumer, producer, or publisher side.

DMP offers support for extendability since new primitive functions can easily be added. However, since primitive functions are very fine-grained and since DMP lacks a blueprint architecture that guides developers in composing an arbitrary set of functionality, application development is more complex and may imply considerable risks for software errors.

DMP partially supports modifiability since primitive functions may have dependencies on each other, which prevents replacing them with other implementations or functions. In addition, typical modifications seem to focus on functional concerns, for instance by replacing a primitive function, and not on the behavior of the system. To the best of our knowledge, it is for instance highly difficult (if not impossible) in DMP to modify a global logging policy by a local change.

In our opinion, the presented DRM software architecture is complementary to an interface specification as presented by DMP. The latter defines how each individual service (i.e. primitive function) can be used by specifying its interfaces, while the former defines how these services can be composed into an overall DRM system by offering a blueprint architecture.

\section{CONCLUSION}

This paper has presented a detailed DRM architecture that provides an answer to three main challenges in DRM: availability of a common core architecture, which can easily be customized to varying and diverse requirements, and which supports the key architectural drivers (interoperability, modifiability, and extendability). The architecture consists of three component groups which offer (1) core services, (2) representation and interpretation services, and (3) security services. The paper has incrementally presented the architecture from three key perspectives, starting from the core (content handling), and then extending it with consumer tracking, and content securing. By way of evaluation, the paper has assessed to what extent three key requirements are supported by the architecture.

With respect to future work, we would like to relate the architecture back to state-of-the-art DRM systems, and use it to implement a proof-of-concept prototype. Since our research started by studying state-of-the-art DRM systems and structuring their functionality in a generic software architecture, we are confident that it is sufficiently detailed to enable the creation and management of DRM systems and content distribution applications. To the best of our knowledge, no similar research results have been published so far.

\section{ACKNOWLEDGEMENTS}

Research for this paper was sponsored by IBBT, the Interdisciplinary institute for BroadBand Technology, and conducted in the context of the E-paper project. The authors are very grateful to Ann Heylighen for her valuable comments on the paper and for proof reading the text.

\section{REFERENCES}

[1] Automating Production of Cross Media Content for Multi-channel Distribution (AXMEDIS), 2006. http://www.axmedis.org/.

[2] Software Architecture Glossary, May 2006 http://www.sei.cmu.edu/architecture/glossary.html.

[3] L. Bass, P. Clements, and R. Kazman. Software Architecture in Practice. Addison-Wesley Longman Publishing Co., Inc., Boston, MA, USA, 1998.

[4] E. Becker, W. Buhse, D. Günnewig, and N. Rump, editors. Digital Rights Management - Technological, Economic, Legal and Political Aspects, volume 2770 of Lecture Notes in Computer Science. Springer, 2003.

[5] L. Chiariglione. Digital Media Project (DMP), 2006. http://www.dmpf.org.

[6] P. Gutmann. Cryptlib Security Toolkit, Sept. 2005. ftp://ftp.franken.de/pub/crypt/cryptlib/manual.pdf.

[7] P. A. Jamkhedkar and G. L. Heileman. DRM as a layered system. In DRM '04: Proceedings of the 4th ACM workshop on Digital Rights Management (DRM 2004), pages 11-21, New York, NY, USA, 2004. ACM Press.

[8] P. A. Jamkhedkar and G. L. Heileman. DRM Interoperability Analysis from the Perspective of a Layered Framework. In DRM '05: Proceedings of the 5th ACM workshop on Digital Rights Management (DRM 2005), pages 17-26, Alexandria, VA, USA, 2005. ACM Press.

[9] S. Michiels, K. Buyens, K. Verslype, W. Joosen, and B. D. Decker. DRM interoperability and reusability through a generic software. INDICARE Monitor - About Consumer and User Issues of Digital Rights, 2(11):1620, Jan. 2006.

[10] S. Michiels, K. Verslype, W. Joosen, and B. D. Decker. Towards a Software Architecture for DRM. In In Proceedings of 5th ACM Workshop on Digital Rights Management (DRM2005), Nov. 2005.

[11] W. Rosenblatt, W. Trippe, and S. Mooney. Digital Rights Management: Business and Technology, chapter 5. Hungry Minds, Inc., 2001.

[12] IBBT e-paper project, 2006 https://projects.ibbt.be/epaper/.

[13] K. Buyens, S. Michiels, and W. Joosen. A refined architecture for DRM. Technical report, K.U.Leuven, Jun. 2006. http://www.cs.kuleuven.be/publicaties/rapporten/cw/CW450.pdf

[14] X. Wang, T. Demartini, B. Wragg, M. Paramasivam, and C. Barlas. The MPEG-21 Rights Expression Language and Rights Data Dictionary. Multimedia, IEEE Transactions on, 7(3):408-417, Jun. 2005. 